\title{
Online Algorithm for Velocity Estimation in Ultrasonic Doppler Measurement
}

\author{
Enyao Shang, Jinchun $\mathrm{Hu}^{*}$ and Shengwu $\mathrm{Du}$ \\ State Key Laboratory of Tribology, Department of Mechanical Engineering, Tsinghua University, Beijing 100084, China \\ ${ }^{*}$ Corresponding author
}

\begin{abstract}
Ultrasonic Doppler technique is widely used for velocity estimation in medical electronic systems. This technique is signal-processing intensive with an increasing requirement on accuracy, real time and stability. The clutter caused by human tissues contains little information about blood flow velocity. Since conventional algorithms are based on an idealized signal model where the residual clutter is not fully considered, highperformance filters are required for clutter rejection in ultrasonic Doppler measurement. This paper focuses on how to extract the velocity information from ultrasonic echo signals without extra filters. We presented an online algorithm that identified the clutter as unknown parameters instead of filtering. The algorithm does not rely on the performance of extra clutter filters. Simulation results show that this algorithm is simpler and more stable than conventional correlation algorithms.
\end{abstract}

Keywords_online algorithm; velocity estimation; ultrasonic; Doppler; filter

\section{INTRODUCTION}

Ultrasonic Doppler technique is widely used in clinical diagnoses, such as cardiology, gynecology and obstetrics. The cardiovascular disease is the leading cause of death that threatens human health. A common symptom of the cardiovascular disease is blood flow abnormality. As one of the most suitable methods for measuring blood flow velocity, ultrasonic Doppler technique has advantages of low cost, no wound and large detection depth. With the rapid development of computer technology and electronic engineering, highprecision, real-time and stable processing has become possible. However, how to extract the velocity information from massive data effectively is still a difficult problem in ultrasonic Doppler measurement.

The conventional algorithms such as auto-correlation algorithm [1], cross-correlation algorithm [2] and maximum likelihood algorithm [3] are based on an idealized signal model. In this model, the residual clutter is not fully considered. The clutter caused by human tissues contains little information about blood flow velocity. High-performance filters are required for clutter rejection. Common examples of linear timeinvariant filters were applied, such as finite impulse response filters [4], infinite impulse response filters [5] and autoregression filters [6]. Besides these, many efforts have been made to improve the filtering. C. Demene et al. [7] presented a method for ultrafast ultrasonic imaging based on spatiotemporal singular value decomposition. The method significantly enhanced image contrast. Noting the different spatial characteristics of human tissues and blood flow, C. H.
$\mathrm{Yu}$ et al. [8] proposed an eigen-based filtering method using Hankel singular value decomposition formulation. The method provided less bias than auto-correlation algorithm. To remove the zero-frequency component, Y. Zhang et al. [9] presented a filter based on empirical mode decomposition. It was proved that the filter had little loss of low blood flow information. Z. Shen et al. [10] suggested a filtering method based on ridge ensemble empirical mode decomposition. The method achieved a high blood-to-clutter energy ratio. Considering the timevarying characteristics of the clutter, various adaptive strategies were proposed [11], [12]. G. Park et al. [13] provided an adaptive method for clutter rejection based on spectral decomposition and tissue acceleration. H. Takahashi et al. [14] presented an adaptive moving-target-indicator filter for clutter rejection. The filter could significantly improve automated identification of the heart wall. In these methods, the clutter was regarded as the interference that should be removed. However, even idealized filters cannot remove the clutter completely due to spectral similarities of the tissue motion and low blood flow. Furthermore, the phase and magnitude of the blood flow signal are changed by filter. The changes will inevitably affect the subsequent processing.

In this paper, we present an online algorithm that identifies the clutter as unknown parameters instead of filtering. The algorithm does not rely on the performance of extra clutter filters. There are three main sections in this paper. Firstly, a typical model of ultrasonic echo signals will be described. Then, we will detail the principle and implementation of the new algorithm. Finally, the algorithm will be evaluated by simulation.

\section{SignAL MODEL}

To extract the velocity information from ultrasonic echo signals, it is necessary to analyze the quantitative relation between them. In this section, we will describe a typical model of the received signal.

The blood flow signal of interest mainly results from the scattering of red blood cells. This signal is so weak that it is easily obscured by the clutter and observation noise. We model the received signal $x(t)$ as

$$
x(t)=s_{\mathrm{b}}(t)+s_{\mathrm{c}}(t)+\varepsilon(t)
$$

where $s_{\mathrm{b}}(t)$ is the blood flow signal with a frequency shift due to Doppler effect, $s_{\mathrm{c}}(t)$ is the clutter and $\varepsilon(t)$ is observation 
noise. A common approach is assuming that the clutter is caused by static tissues [15]. Therefore, the clutter has the same frequency as the excitation. A typical excitation in ultrasonic Doppler measurement is periodic pulses. Due to the use of digital processing system, a discrete-time data set can be sampled. Let $x\left[n_{\mathrm{s}}, n_{\mathrm{prf}}\right], s_{\mathrm{b}}\left[n_{\mathrm{s}}, n_{\mathrm{prf}}\right], s_{\mathrm{c}}\left[n_{\mathrm{s}}, n_{\mathrm{prf}}\right]$ and $\varepsilon\left[n_{\mathrm{s}}, n_{\mathrm{prf}}\right]$ denote the values of $x(t), s_{\mathrm{b}}(t), s_{\mathrm{c}}(t)$ and $\varepsilon(t)$ at the $n_{\mathrm{s}}$-th sampling moment in the $n_{\text {prr-th }}$ theriod. If the excitation pulses are sinusoidal, then

$$
s_{\mathrm{b}}\left[n_{\mathrm{s}}, n_{\mathrm{prf}}\right] \approx A_{\mathrm{b}} \sin \left(2 \pi f_{0} T_{\mathrm{s}} n_{\mathrm{s}}-4 \pi f_{0} T_{\mathrm{prf}} n_{\mathrm{prf}} v / c+\varphi_{\mathrm{b}}\right),
$$

$$
s_{\mathrm{c}}\left[n_{\mathrm{s}}, n_{\mathrm{prf}}\right] \approx A_{\mathrm{c}} \sin \left(2 \pi f_{0} T_{\mathrm{s}} n_{\mathrm{s}}+\varphi_{\mathrm{c}}\right),
$$

where $A_{\mathrm{b}}$ and $A_{\mathrm{c}}$ represent the amplitude attenuation due to propagation losses, $\varphi_{\mathrm{b}}$ and $\varphi_{\mathrm{c}}$ are the phase shifts that are related to the detection depths, $f_{0}$ is the center frequency of the transmitted pulses, $T_{\mathrm{s}}$ is the sampling interval, $T_{\mathrm{prf}}$ is the pulse repetition period, $v$ is the blood flow velocity, and $c$ is the speed of sound.

Finally, the sampling value could be expressed by

$$
\begin{aligned}
x\left[n_{\mathrm{s}}, n_{\mathrm{prr}}\right] & =s_{\mathrm{b}}\left[n_{\mathrm{s}}, n_{\mathrm{prf}}\right]+s_{\mathrm{c}}\left[n_{\mathrm{s}}, n_{\mathrm{prf}}\right]+\varepsilon\left[n_{\mathrm{s}}, n_{\mathrm{prf}}\right] \\
& \approx A_{\mathrm{b}} \sin \left(2 \pi f_{0} T_{\mathrm{s}} n_{\mathrm{s}}-4 \pi f_{0} T_{\mathrm{prf}} n_{\mathrm{prf}} v / c+\varphi_{\mathrm{b}}\right) \\
& +A_{\mathrm{c}} \sin \left(2 \pi f_{0} T_{\mathrm{s}} n_{\mathrm{s}}+\varphi_{\mathrm{c}}\right)+\varepsilon\left[n_{\mathrm{s}}, n_{\mathrm{prf}}\right] .
\end{aligned}
$$

In summary, we model the sampling value as the summation of the blood flow signal, the clutter and observation noise. The blood flow signal has a frequency shift that is proportional to the velocity due to Doppler effect.

\section{Signal Processing Algorithm}

The previous section analyzed the quantitative relation between the blood flow velocity and the received signal. The problem now is how to estimate the velocity $v$ based on 2-D data set $x\left[n_{\mathrm{s}}, n_{\mathrm{prr}}\right]$. This section will detail the principle and implementation of the new algorithm.

\section{A. Algorithm Principle}

We wish to maximize the probability density function $p(x ; v)$ over $v$. Consider the velocity estimator

$$
\hat{v}=\underset{v}{\arg \max }[p(x ; v)] .
$$

Assume that $\varepsilon\left[n_{\mathrm{s}}, n_{\mathrm{prf}}\right]$ is white Gaussian noise. Then,

$$
\frac{1}{N_{\mathrm{s}}} \sum_{n_{\mathrm{s}}=0}^{N_{\mathrm{s}}-1} \varepsilon\left[n_{\mathrm{s}}, n_{\mathrm{prf}}\right] \exp \left(\mathrm{i} 2 \pi f_{0} T_{\mathrm{s}} n_{\mathrm{s}}\right)
$$

is also white Gaussian noise. Let $\sigma^{2}$ denote the variance of $\varepsilon\left[n_{\mathrm{s}}, n_{\mathrm{prf}}\right]$ and

$$
J=\sum_{n_{\mathrm{prf}}=0}^{N_{\mathrm{prf}}-1}\left|\frac{1}{N_{\mathrm{s}}} \sum_{n_{\mathrm{s}}=0}^{N_{\mathrm{s}}-1} \varepsilon\left[n_{\mathrm{s}}, n_{\mathrm{prf}}\right] \exp \left(\mathrm{i} 2 \pi f_{0} T_{\mathrm{s}} n_{\mathrm{s}}\right)\right|^{2} .
$$

Thus, the probability density function can be written as

$$
p(x ; v)=\left(\pi \sigma^{2} / N_{\mathrm{s}}\right)^{-N_{\mathrm{prf}} / 2} \exp \left(-N_{\mathrm{s}} J / \sigma^{2}\right) .
$$

Now we are equivalently minimizing $J$ in maximizing $p(x ; v)$. From $(5)$,

$$
\hat{v}=\underset{v}{\arg \max }[p(x ; v)]=\underset{v}{\arg \min }(J) .
$$

Let $f=2 v f_{0} T_{\text {prf }} / c, \theta_{1}=0.5 A_{\mathrm{b}} \cos \varphi_{\mathrm{b}}, \theta_{2}=0.5 A_{\mathrm{b}} \sin \varphi_{\mathrm{b}}$, $\theta_{3}=0.5 A_{\mathrm{c}} \cos \varphi_{\mathrm{c}}, \theta_{4}=0.5 A_{\mathrm{c}} \sin \varphi_{\mathrm{c}}$ and

$$
y[n]=\frac{1}{N_{\mathrm{s}}} \sum_{n_{\mathrm{s}}=0}^{N_{\mathrm{s}}-1} x\left[n_{\mathrm{s}}, n\right] \exp \left(\mathrm{i} 2 \pi f_{0} T_{\mathrm{s}} n_{\mathrm{s}}\right) .
$$

The real part and imaginary part of $y[n]$ are denoted by $y_{\mathrm{R}}[n]$ and $y_{\mathrm{I}}[n]$ respectively. Then, we have

$$
\begin{aligned}
& J \approx \sum_{n=0}^{N_{\text {prf }}-1}\left(y_{\mathrm{R}}[n]-\theta_{4}+\theta_{1} \sin (2 \pi f n)-\theta_{2} \cos (2 \pi f n)\right)^{2}+ \\
& \sum_{n=0}^{N_{\text {prf }}-1}\left(y_{\mathrm{I}}[n]-\theta_{3}-\theta_{1} \cos (2 \pi f n)-\theta_{2} \sin (2 \pi f n)\right)^{2} .
\end{aligned}
$$

$J$ is a function of $f, \theta_{1}, \theta_{2}, \theta_{3}$ and $\theta_{4} . f$ is proportional to the velocity. Since $J(f+1)=J(f)$, we only need to consider $-0.5<f \leq 0.5$. Besides $f$ of interest, $\theta_{1}, \theta_{2}, \theta_{3}$ and $\theta_{4}$ are all unknown parameters. Consider the parameters that minimize $J$, denoted by

$$
\hat{\boldsymbol{\theta}}=\left[\begin{array}{llll}
\hat{\theta}_{1} & \hat{\theta}_{2} & \hat{\theta}_{3} & \hat{\theta}_{4}
\end{array}\right]^{\mathrm{T}}
$$

The superscript $\mathrm{T}$ represents matrix transpose.

Setting the partial derivatives of $J$ to zero, we have

$$
\left.\frac{\partial J}{\partial \theta}\right|_{\hat{\theta}}=0
$$

That is

$$
\left(\begin{array}{cccc}
N_{\mathrm{prf}} & 0 & \sum \cos -\sum \sin \\
0 & N_{\mathrm{prf}} & \sum \sin \sum_{\cos } \\
\sum \cos & \sum \sin & N_{\text {prf }} & 0 \\
-\sum \sin & \sum \cos & 0 & N_{\text {prf }}
\end{array}\right) \hat{\boldsymbol{\theta}}=\left(\begin{array}{c}
\sum y_{\mathrm{I}} \cos -\sum y_{\mathrm{R}} \sin \\
\sum y_{\mathrm{I}} \sin +\sum y_{\mathrm{R}} \cos \\
\sum y_{\mathrm{I}} \\
\sum y_{\mathrm{R}}
\end{array}\right)
$$




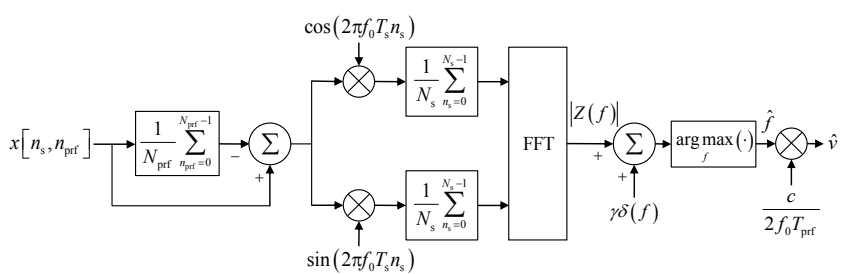

FIGURE I. SCHEMATIC DIAGRAM OF BATCH ALGORITHM.

If $f \neq 0$, then solving (14) yields $\hat{\theta}$. Substituting $\hat{\theta}$ in and letting $z[n]=y[n]-\sum y[n] / N_{\text {prf }}$, we have

$$
\left.J\right|_{\hat{\theta}}=\sum|z[n]|^{2}-\frac{1}{N_{\text {prf }}} \frac{\left|\sum z[n] \exp (-\mathrm{i} 2 \pi f n)\right|^{2}}{1-\left|\frac{1}{N_{\text {prf }}} \sum \exp (-\mathrm{i} 2 \pi f n)\right|^{2}} .
$$

Let $Z(f)$ denote the Fourier transformation of $z[n]$. If $N_{\text {prf }}$ is large enough, then

$$
\left.J\right|_{\hat{\theta}} \approx \sum|z[n]|^{2}-|Z(f)|^{2} / N_{\text {prf }}
$$

From (9),

$$
\begin{aligned}
\hat{v} & =\underset{v}{\arg \min }(J)=\frac{c}{2 f_{0} T_{\text {prf }}} \underset{f}{\arg \min }(J) \\
& =\frac{c}{2 f_{0} T_{\text {prf }}} \underset{f}{\arg \min }\left(\left.J\right|_{\hat{\theta}}\right)=\frac{c}{2 f_{0} T_{\text {prf }}} \underset{f}{\arg \max }|Z(f)| .
\end{aligned}
$$

However, the estimator is not always feasible because (15) requires $f \neq 0$. Noting that $|Z(0)|=\sum z[n]=0$, the estimator is invalid if $f \approx 0$. Hence, revise(17) as

$$
\hat{v}=\frac{c}{2 f_{0} T_{\text {prf }}} \underset{f}{\arg \max }(|Z(f)|+\gamma \delta(f)),
$$

where $\gamma$ is a constant selected by user and $\delta$ is the Dirac function.

In summary, the velocity estimator of the new algorithm is

$$
\hat{v}=\frac{c}{2 f_{0} T_{\text {prf }}} \arg \max (|Z(f)|+\gamma \delta(f)),
$$

where $c$ is the speed of sound, $f_{0}$ is the center frequency of transmitted pulses, $T_{\text {prf }}$ is the pulse repetition period, $\gamma$ is a constant selected by user, $\delta$ is the Dirac function, $Z(f)$ is the Fourier transform of $z[n]$ and

$$
z[n]=\frac{1}{N_{\mathrm{s}}} \sum_{n_{\mathrm{s}}}\left[\left(x\left[n_{\mathrm{s}}, n\right]-\frac{1}{N_{\text {prf }}} \sum_{n} x\left[n_{\mathrm{s}}, n\right]\right) \exp \left(\mathrm{i} 2 \pi f_{0} T_{\mathrm{s}} n_{\mathrm{s}}\right)\right]
$$

\section{B. Algorithm Implementation}

The above batch algorithm processes all the data at once. It means that users have to wait for all the available data, or compute the preceding data repeatedly. To reduce time and calculation work, we process the samples sequentially in time.

If we received the data of the $k$-th period just now, then compute $y[k-1]$ based on the new data $x\left[n_{\mathrm{s}}, k-1\right]$ by

$$
y[k-1]=\frac{1}{N_{\mathrm{s}}} \sum_{n_{\mathrm{s}}=0}^{N_{\mathrm{s}}-1} x\left[n_{\mathrm{s}}, k-1\right] \exp \left(\mathrm{i} 2 \pi f_{0} T_{\mathrm{s}} n_{\mathrm{s}}\right)
$$

Introduce intermediate variables $\bar{y}^{(k)}$ and $u^{(k)}$. Let

$$
\bar{y}^{(k)}=\frac{1}{k} \sum_{n=0}^{k-1} y[n]
$$

$$
u^{(k)}=\frac{1}{k} \sum_{n=0}^{k-1} \exp (-\mathrm{i} 2 \pi f n)
$$

Then, their recursive formulas are

$$
\begin{aligned}
\bar{y}^{(k)} & =\frac{1}{k} \sum_{n=0}^{k-1} y[n]=\frac{1}{k}\left(\sum_{n=0}^{k-2} y[n]+y[k-1]\right) \\
& =\bar{y}^{(k-1)}+\frac{1}{k}\left(y[k-1]-\bar{y}^{(k-1)}\right)
\end{aligned}
$$

$$
\begin{aligned}
u^{(k)} & =\frac{1}{k} \sum_{n=0}^{k-1} \exp (-\mathrm{i} 2 \pi f n) \\
& =u^{(k-1)}+\frac{1}{k}\left[\exp (-\mathrm{i} 2 \pi f(k-1))-u^{(k-1)}\right] .
\end{aligned}
$$

Letting $Z^{(k)}(f)$ denote $Z(f)$ obtained from the first $\mathrm{k}$ periods, we have

$$
\begin{gathered}
Z^{(k)}(f)=Z^{(k-1)}(f)+\frac{k-1}{k}\left[y[k-1]-\bar{y}^{(k-1)}\right] \\
{\left[\exp (-\mathrm{i} 2 \pi f(k-1))-u^{(k-1)}\right] .}
\end{gathered}
$$

Summarize the recursive procedures as follows:

Step 1. Initialize the variables: $k=0, \bar{y}^{(0)}=0, u^{(0)}=0, Z^{(0)}(f)=0$;

Step 2. Increase the index $k$ by 1 and wait for new data; 
Step 3. Compute $y[k-1]$ based on the most recent data $x\left[n_{\mathrm{s}}, k-1\right]$ by $(21)$;

Step 4. Update the variables $\bar{y}^{(k)}, u^{(k)}$ and $Z^{(k)}(f)$ by $(24)(25)(26)$;

Step 5. Compute $\left|Z^{(k)}(f)\right|$ and select a proper $\gamma$;

Step 6. Compute $\hat{v}^{(k)}$ by (19), and then return to Step 2 until no more data are sampled.

\section{SimUlation Results AND Discussion}

In this section, we will compare the new algorithm with conventional correlation algorithms by simulation. Simulate echo signals with the toolkit Field II, and then compute the velocity estimators separately.

Firstly, set sound field parameters. The speed of sound in human tissues is $1540 \mathrm{~m} / \mathrm{s}$. The excitation is sinusoidal periodic pulses with the center frequency of $5 \mathrm{MHz}$. There are 32 pulses with intervals of $100 \mu \mathrm{s}$. The sampling interval is $25 \mathrm{~ns}$. The radius of the vessel is $4 \mathrm{~mm}$ and the vessel is at the depth of $30 \mathrm{~mm}$ with a $45^{\circ}$ angle to the ultrasonic beam. A 128 -element ultrasonic phased array probe is used. The elements are $0.15 \mathrm{~mm}$ width and $5 \mathrm{~mm}$ length with kerfs of $0.03 \mathrm{~mm}$. The intensity ratio of red blood cells to static tissues is 0.1 . The given velocity $v$ has a parabolic profile where the maximum is $0.8 \mathrm{~m} / \mathrm{s}$. The algorithms are implemented in the MATLAB programs. 58,062 points are generated using the Monte Carlo method. The simulation results are shown in Figure 2.

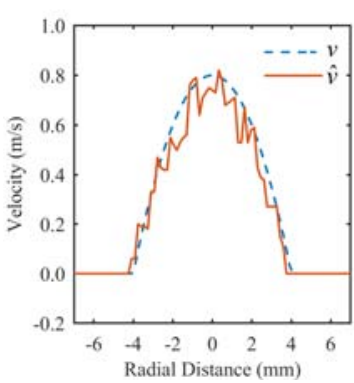

(A)

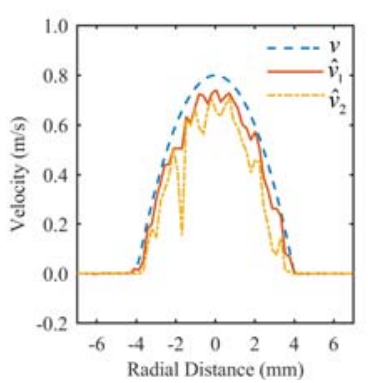

(B)

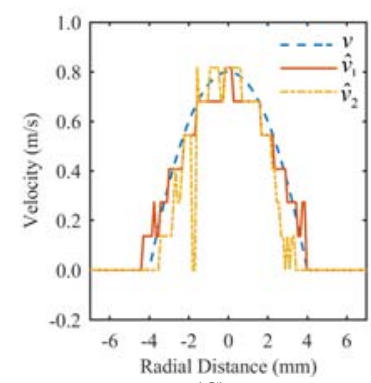

(C)

FIGURE II. VELOCITY ESTIMATORS OF (A) THE NEW ALGORITHM, (B) AUTO-CORRELATION ALGORITHM AND (C) CROSSCORRELATION ALGORITHM.

In Figure 2, the $x$-axis shows the radial distance from the center of the vessel. In Figure 2(A), $v$ represents the theoretical velocity and $\hat{v}$ represents the velocity estimator of the new algorithm. Although $v$ is slightly smaller than $\hat{v}$, the new algorithm performs well. In Figure 2(B), a second-order lowpass filter and a second-order Chebyshev Type I high-pass filter are used. $\hat{v}_{1}$ and $\hat{v}_{2}$ represent the velocity estimators of auto-correlation algorithm. $\hat{v}_{1}$ is calculated with proper filter parameters, while $\hat{v}_{2}$ is calculated with improper filter parameters. Similarly, in Figure 2(C), a second-order Chebyshev Type I high-pass filter is used. $\hat{v}_{1}$ and $\hat{v}_{2}$ represent the velocity estimators of cross-correlation algorithm. From Figure 2(B) and Figure 2(C), both the correlation algorithms provide good performance with proper filters, but produce notable error when the filters are improper. In other words, correlation algorithms rely on the performance of clutter filters. Auto-correlation algorithm employs a low-pass filter to remove high-frequency noise and a high-pass filter to the clutter. Crosscorrelation algorithm requires a high-pass filter that is essential in noisy environment. By contrast, the new algorithm is simpler because it does not involve the design of extra filters.

In summary, we compare the algorithms in three aspects.

1) Accuracy. All the algorithms work well.

2) Stability. Correlation algorithms are sensitive to the clutter. The new algorithm which does not rely on the performance of extra filters is the most stable one.

3) Simplicity. Compared with the conventional correlation algorithms, the new algorithm is easier to implement.

\section{CONCLUSION}

This paper focused on how to extract the velocity information from ultrasonic echo signals without extra filters.

An online algorithm was presented. Since the algorithm identifies the clutter as unknown parameters instead of filtering, it does not rely on the performance of extra clutter filters. To reduce time and calculation work, the sequential processing is recommended.

Compared with the conventional correlation algorithms, the new algorithm is simpler and more stable.

\section{ACKNOWLEDGMENT}

We thank Dr. Yu Zhu and Dr. Ming Zhang for helpful conversations, Dr. Kaiming Yang for excellent technical assistance, Zhongcheng Zhou and Peng Lei for assistance with the experiments.

\section{REFERENCES}

[1] A. C. Chan, E. Y. Lam and V. J. Srinivasan, "Comparison of Kasai autocorrelation and maximum likelihood estimators for Doppler optical coherence tomography", IEEE Trans. Med. Imag., vol. 32, no. 6, pp. 1033-1042, Jun. 2013.

[2] J. A. Jensen, S. I. Nikolov, A. C. H. Yu and D. Garcia, "Ultrasound vector flow imaging-Part I: Sequential systems", IEEE Trans. Ultrason. Ferroelect. Freq. Control, vol. 63, no. 11, pp. 1704-1721, Nov. 2016.

[3] N. Thong-un, W. Wongsaroj, W. Treenuson, J. Chanwutitum and H. Kikura, "Doppler frequency estimation using maximum likelihood function for low ultrasonic velocity profile", Acoust. Sci. \& Tech., vol. 38, no. 5, pp. 268-271, 2017.

[4] L. W. Chang, K. H. Hsu and P. C. Li, "Graphics processing unit-based high-frame-rate color doppler ultrasound processing," IEEE Trans. 
Ultrason. Ferroelect. Freq. Contr., vol. 56, no. 9, pp. 1856-1860, Sep. 2009.

[5] S. Koçkanat, T. Koza and N. Karaboğa, "IIR filter paramater estimation with ABC algorithm for mitral valve Doppler signal denoising," 2015 23nd Signal Processing and Communications Applications Conference (SIU), Malatya, 2015, pp. 1989-1992.

[6] J. Kang, Y. Kim, W. Lee and Y. Yoo, "A new dynamic complex baseband pulse compression method for chirp-coded excitation in medical ultrasound imaging", IEEE Trans. Ultrason. Ferroelect. Freq. Contr., vol. 64, no. 11, pp. 1698-1710,Nov. 2017.

[7] C. Demene, T. Deffieux, M. Pernot, B. F. Osmanski, V. Biran, J. L. Gennisson et al., "Spatiotemporal clutter filtering of ultrafast ultrasound data highly increases Doppler and fultrasound sensitivity", IEEE Transactions on Medical Imaging, vol. 34, no. 11, pp. 2271-2285, Nov. 2015.

[8] C. H. Yu and R. S. C. Cobbold, "Single-ensemble-based eigenprocessing methods for color flow imaging - Part I. The Hankel-SVD filter", IEEE Trans. Ultrason. Ferroelect. Freq. Contr., vol. 55, no. 3, pp. 559-572, Mar. 2008.

[9] Y. Zhang, Y. Gao, L. Wang, J. Chen and X. Shi, "The removal of wall components in Doppler ultrasound signals by using the empirical mode decomposition algorithm", IEEE Trans. Biomed. Eng., vol. 54, no. 9, 2007, pp. 1631-1642.

[10] Z. Shen, N. Feng, Y. Shen, and C. H Lee, "A ridge ensemble empirical mode decompositi on approach to clutter rejection for ultrasound color flow imaging", IEEE Trans. Biomed. Eng., vol. 60, no. 6, pp. 1477-1487, 2013.

[11] G. Park, S. Yeo, J. J. Lee, C. Yoon, H. Koh, H. Lim, Y. Kim, H. Shim and Y. Yoo, "New adaptive clutter rejection based on spectral analysis for ultrasound color Doppler imaging: Phantom and in vivo abdominal study", IEEE Trans. Biomed. Eng., vol. 61, no. 1, pp. 55-63, Jan. 2014.

[12] C. H. Yu and L. Lovstakken, "Eigen-based clutter filter design for ultrasound color flow imaging: A review", IEEE Trans. Ultrason. Ferroelect. Freq. Contr., vol. 57, no. 5, pp. 1096-1111, May 2010.

[13] G. Park, Y. Kim, H. Shim, H. W. Koh, H. Lim, J. J. Lee, S. Yeo, T. K. Song and Y. Yoo, "New adaptive clutter rejection based on spectral decomposition and tissue acceleration for ultrasound color Doppler imaging." IEEE Ultrason. Symp., 2014:1484-1487.

[14] H. Takahashi, H. Hasegawa and H. Kanai, "Improvement of automated identification of the heart wall in echocardiography by suppressing clutter component", Japanese Journal of Applied Physics, vol. 52, no. 52, pp. 1044-1055, 2013.

[15] D. H. Evans and W. N. McDicken, "Doppler Ultrasound: Physics Instrumentation and Signal Processing", New York, NY:Wiley, 2000. 